\title{
Doença celíaca: sua relação com a saúde bucal
}

\section{Celiac disease's relationship with the oral health}

\author{
Michelle Soares RAUEN ${ }^{1}$ \\ Jacqueline Camilli de Vasconcellos BACK' \\ Emília Addison Machado MOREIRA²
}

\section{R E S U M O}

Doença Celíaca é uma intolerância permanente às proteínas contidas no glúten de alguns cereais, como o trigo, o centeio, a cevada e a aveia. A doença manifesta-se principalmente nos primeiros dois anos de vida, sendo o intestino delgado o principal órgão afetado, com manifestações clínicas de diarréia, vômitos e emagrecimento; porém, o diagnóstico, muitas vezes, é difícil, devido ao grande número de casos atípicos da doença. Nestes casos, os sintomas podem ser numerosos e diversificados, tais como baixa estatura, anemia, osteoporose, hipoplasia do esmalte dentário, além de sintomas próprios do quadro clínico de outras doenças imunológicas que podem associar-se à doença celíaca, tais como diabetes mellitus, dermatite herpertiforme, doenças da tireóide, alergia, estomatite aftosa recorrente, entre outras. Devido a essa associação, os profissionais da saúde procurados pelos pacientes podem não relacionar os sintomas à enteropatia; entretanto, esta, se não tratada, pode trazer várias outras complicações à saúde. O objetivo desta comunicação é demonstrar a importância das manifestações bucais, as quais, quando devidamente observadas, contribuem ao diagnóstico da doença celíaca.

Termos de indexação: doença celíaca, hipoplasia do esmalte dentário, manifestações bucais, saúde bucal.

\section{A B S T R A C T}

Celiac Disease is a permanent intolerance to proteins contained in the gluten of some cereals, such as wheat, rye, barley and oat. The disease appears mainly during the first two years of life, the small bowel being the main affected organ, with clinical manifestations such as diarrhea, vomiting and weight loss. The diagnosis, however, is often difficult, due to the large number of atypical manifestations of the disease. In such cases, numerous and diversified symptoms, such as low stature, anemia, osteoporosis, and dental enamel hypoplasia, may be concurrent with symptoms of immune diseases associated to the celiac disease (diabetes mellitus, dermatitis herpetiformis, thyroid diseases, allergy, and recurrent aphtous stomatitis, among others). With such vast array of symptoms, patients will seek health professionals, who, however, might have trouble relating the symptoms to the celiac disease. If this illness is not treated properly, chances are that several other health complications will show up. The objective of this communication is to demonstrate that oral manifestations are important contributors to the diagnosis of the celiac disease.

Indexing terms: celiac disease, dental enamel hypoplasia, oral manifestations, oral health.

\footnotetext{
1 Programa de Pós-Graduação em Odontologia, Universidade Federal de Santa Catarina. Campus Universitário, Trindade, 88040-970, Florianópolis, SC, Brasil. Correspondência para/Correspondence to: M. SOARES. E-mail: $<$ michelle_soares@yahoo.com.br>.

2 Programa de Pós-Graduação em Odontologia, Universidade Federal de Santa Catarina. Florianópolis, SC, Brasil. E-mail: <addison@ccs.ufsc.br>.
} 


\section{N T R O D U Ç Ã O}

Doença celíaca (DC) é uma intolerância permanente ao glúten, presente em alguns cereais; denominada também de enteropatia glúten-sensível, caracteriza-se por atrofia total ou subtotal das vilosidades do intestino delgado proximal, levando, conseqüentemente, à má absorção da grande maioria dos nutrientes. A doença pode atingir pessoas de qualquer idade ${ }^{1} \mathrm{e}$ sua manifestação depende não só do uso de glúten na dieta, mas também, da presença de fatores genéticos, imunológicos e ambientais ${ }^{2,3}$.

A prevalência da doença é muito variável de país para país ${ }^{4}$, sendo desconhecidos dados estatísticos oficiais no Brasil. Atinge predominantemente os indivíduos de cor branca, mas no Brasil, devido à alta miscigenação racial, já foi descrita em mulatos. Manifesta-se geralmente, a partir do segundo semestre de vida, coincidindo com a introdução dos cereais na alimentação².

O diagnóstico de DC deve ser baseado em três pilares: o exame clínico, por meio de exame físico e anamnese detalhada, além da análise histopatológica do intestino delgado, e dos marcadores séricos ${ }^{5,6}$. O diagnóstico final deve sempre basear-se na biópsia7,8, a qual revela a mucosa anormal do intestino delgado proximal, com as vilosidades atrofiadas ou ausentes, aumento no comprimento das criptas e no número de linfócitos intra-epiteliais ${ }^{3,9}$.

O único tratamento possível e eficaz para DC, em todas as formas clínicas, é o dietético, devendo-se excluir o glúten da alimentação durante toda a vida ${ }^{8}$, o que leva à remissão dos sintomas e restauração da morfologia normal da mucosa ${ }^{3,4}$. O glúten, presente nos cereais trigo, centeio, cevada e aveia, deve ser substituído pelo milho, arroz, batata e mandioca, sendo considerados alimentos permitidos os grãos, gorduras, óleos e azeites, legumes, hortaliças, frutas, ovos, carnes e leite, lembrando sempre que a dieta deverá atender às necessidades nutricionais de acordo com a idade do indivíduo ${ }^{10}$. Além da dieta, o paciente celíaco deve estar atento também à composição dos medicamentos prescritos para ele ${ }^{11}$. Segundo Sdepanian et al. ${ }^{12}$, quanto maior o grau de conhecimento da doença e de seu tratamento, maior a obediência à dieta desprovida de glúten.

Quanto ao prognóstico, há a possibilidade de uma série de complicações da DC quando não tratada, como esterilidade, osteoporose, endocrinopatias, distúrbios neurológicos e psiquiátricos ${ }^{13}$, doenças hepáticas, doenças do sistema conjuntivo ${ }^{14} \mathrm{e}$ associação com doenças auto-imunes, tais como dermatite herpertiforme, diabetes mellitus², deficiência seletiva de $\lg \mathrm{A}^{15}$ e doenças da tireóide $^{16}$. E, quando comparados à população em geral, estes pacientes estão em maior risco de desenvolver enteropatia associada ao linfoma de célula $T$, ao carcinoma de faringe e esôfago e ao adenocarcinoma de intestino delgado ${ }^{17,18}$.

A DC pode apresentar vários quadros clínicos, com diversos sinais e sintomas. A forma clássica é a que se manifesta nos primeiros anos de vida, com quadro de diarréia crônica, anorexia, vômitos, emagrecimento, comprometimento variável do estado nutricional, irritabilidade, inapetência, déficit de crescimento, dor e distensão abdominal, atrofia da musculatura glútea e palidez por anemia ferropriva ${ }^{10}$.

Nos últimos anos, vem se modificando a apresentação clínica e a idade do diagnóstico da DC, pois seus sintomas, muitas vezes, são inespecíficos e atípicos, principalmente em crianças mais velhas e adultos ${ }^{19}$. Especialmente nas duas últimas décadas, com o advento dos marcadores sorológicos para detectar os anticorpos antigliadina, antireticulina e antiendomísio, presentes na doença celíaca ${ }^{6,7,9}$, os quadros atípicos e assintomáticos estão sendo reconhecidos e acompanhados. Segundo Kotze et al. ${ }^{20}$, a pesquisa dos anticorpos antiendomísio deve ser o teste sorológico de escolha tanto para o diagnóstico, como para o controle da DC, devido à sua alta especificidade e sensibilidade.

DC latente é como se define a condição dos pacientes que apresentam, num dado 
momento, biópsia jejunal normal consumindo glúten; porém, em outro período, podem apresentar atrofia das vilosidades intestinais, as quais voltarão ao normal com a utilização da dieta sem glúten². Já a DC silenciosa é a condição em que os pacientes apresentam biópsia alterada, porém sem sintomatologia ${ }^{3}$.

Nas formas atípicas, os sintomas digestivos estão ausentes ou são pouco relevantes, mas aparecem manifestações isoladas, como anemia por deficiência de ferro refratária à ferroterapia oral, constipação intestinal, osteoporose, esterilidade, baixa estatura ou atraso no crescimento ${ }^{10}$. Lesões na mucosa oral ou defeitos no esmalte dentário podem ser os únicos sinais presentes nos casos atípicos da doença ${ }^{21}$. O objetivo deste trabalho é contribuir ao diagnóstico da Doença Celíaca, demonstrando a importância das manifestações bucais como sintomas dessa enteropatia.

\section{Saúde Bucal e Doença Celíaca}

A Doença Celíaca relaciona-se com a odontologia pelas alterações nas estruturas bucais. A hipoplasia do esmalte dentário, embora pouco destacada na literatura, é um sinal freqüente, bastante comum na forma clínica silenciosa ${ }^{22}$, sendo possivelmente a única manifestação da doença ${ }^{23}$ em crianças e adolescentes celíacos não tratados.

A hipoplasia de esmalte é a anormalidade mais comum no desenvolvimento do esmalte dentário. A lesão é manifestada como um defeito no tecido do esmalte devido a uma injúria às células produtoras, os ameloblastos. Esta injúria pode ter inúmeras causas, geralmente de ordem sistêmica, dentre elas as desordens nutricionais. Clinicamente, o defeito é visto como uma circunferência ou faixa com irregularidades no esmalte, ou com discretas fissuras. A lesão geralmente adquire coloração amarela ou marrom pela deposição de pigmentos extrínsecos ${ }^{24}$.

Segundo alguns autores, a hipoplasia do esmalte tem maior prevalência em pacientes com doença celíaca, quando comparados à população em geral; ademais, tais autores indicam que estas lesões podem constituir indícios para o diagnóstico da doença, principalmente nos casos assintomáticos ${ }^{25-28}$.

Desde 1979, já havia relato de caso clínico de hipoplasia de esmalte em paciente celíaco ${ }^{29}$. Nos últimos tempos, a hipoplasia tem sido observada em pacientes celíacos, como defeitos do esmalte na dentição permanente, distribuídos simétrica e cronologicamente nas quatro hemiarcadas dentárias ${ }^{25}$. Estes foram denominados por Aine ${ }^{23}$ como defeitos no esmalte "tipo-celíaco", com a seguinte classificação: grau 1, defeito na cor do esmalte; grau 2, discreto defeito estrutural com sulcos horizontais típicos; grau 3, defeitos estruturais maiores, com sulcos horizontais profundos e grandes fossas verticais; grau 4, defeito estrutural severo, no qual a forma do dente pode ser modificada.

Na Finlândia, pesquisadores ${ }^{26,27}$ encontraram defeitos simétricos e cronológicos nas quatro arcadas, em $96 \%$ das crianças e $83 \%$ dos adultos com DC; porém, em apenas $4 \%$ no grupo controle saudável. Já na cidade de Londres, foram comparados 42 pacientes adultos com DC, a 18 indivíduos-controle, concluindo-se que defeitos no esmalte, "tipo-celíaco", são pouco freqüentes em adultos celíacos ${ }^{30}$.

$\mathrm{Na}$ Espanha25, o exame bucal em 137 pacientes (5 a 68 anos), com DC diagnosticada por meio de biópsia intestinal, e em 52 pacientes-controle (5 a 64 anos), revelou defeitos no esmalte de $53 \%$ dos pacientes com DC e de $42 \%$ dos pacientes-controle; tais defeitos eram simétricos em $72 \%$ do grupo dos celíacos e em $41 \%$ do grupo controle. Os incisivos e os primeiros molares foram os dentes mais afetados, talvez pela fase da odontogênese coincidir com a fase ativa da doença. Em estudo semelhante, em Roma ${ }^{28}$, realizado apenas com crianças, encontraram-se defeitos no esmalte (opacidade e/ou hipoplasia) em $28 \%$ do grupo celíaco e em $15 \%$ do grupo controle, demonstrando também que há uma correlação entre a idade do 
diagnóstico da DC e o número de dentes afetados, ou seja, com defeitos no esmalte.

Nikiforuk \& Frase ${ }^{24}$ formularam a hipótese de que a hipoplasia do esmalte é causada por hipocalcemia (baixas concentrações de cálcio durante a formação do esmalte); contudo, eles não estudaram pacientes com DC. Segundo Seow $^{31}$, fatores sistêmicos estão associados à hipoplasia do esmalte, tais como a desnutrição, deficiência de vitamina $D$ e $A$ e a hipocalcemia. Para Aine ${ }^{26}$, a ingestão de glúten e as conseqüências disto são responsáveis pelos defeitos de esmalte nos pacientes celíacos. O desenvolvimento dos dentes permanentes se dá basicamente nos primeiros sete anos de vida e, sendo a cronologia dos dentes conhecida ${ }^{32}$, podemos observar a época em que aconteceram os distúrbios, influenciando na formação do esmalte dentário.

Embora existam evidências de que a DC esteja relacionada à hipoplasia do esmalte, ainda há controvérsias quanto à natureza desta relação. Demonstrou-se este fato num estudo realizado em 56 indivíduos, cujo parentesco com pacientes celíacos era de primeiro grau ${ }^{33}$. Submetidos a exame dental e biópsia intestinal, 25 desses parentes apresentaram lesões de esmalte "tipo-celíaco", porém, apenas sete foram diagnosticados como portadores de DC. Os autores formularam a hipótese de que deve ocorrer um processo imunológico induzido pelo glúten, entre os seis meses e sete anos de idade, que afeta o órgão produtor do esmalte, resultando em defeitos na formação do esmalte. Ressalvam os autores que, nestes casos, ou a enteropatia se resolveu espontaneamente, ou essas pessoas são celíacos latentes, pois a biópsia intestinal não apresentou características microscópicas típicas.

Para Aguirre et al. ${ }^{25}$, pacientes que apresentam defeitos no esmalte deveriam ser investigados pela possibilidade de apresentarem DC. Segundo Aine ${ }^{22}$, os profissionais de saúde devem estar atentos, já que, geralmente, as lesões no esmalte dentário são brandas e dificilmente detectáveis.
Em alguns casos, a DC pode contribuir para o desenvolvimento da desnutrição protéico-calórica e esta, dependendo da idade em que se estabeleça, contribui para o aparecimento de alterações na saúde bucal, tais como atraso na erupção dental, diminuição do tamanho dos dentes, problemas na formação do esmalte e disfunção das glândulas salivares ${ }^{33}$.

Kotze ${ }^{2}$, após revisão bibliográfica a respeito de DC, concluiu que os principais sinais bucais da doença são a queilite angular, glossite e língua despapilada. Sabe-se que vermelhidão e dor na língua, com atrofia papilar, estão relacionados às deficiências de vitamina $B_{12}$, de ácido fólico e de ferro, cujas absorções podem ser afetadas pelos efeitos da DC no intestino delgado ${ }^{21}$.

Algumas manifestações da DC na cavidade bucal foram observadas em um estudo ${ }^{21}$ realizado com 128 pacientes celíacos em dieta isenta de glúten, com diagnóstico comprovado por meio de biópsia, além de 30 pacientes-controle. Realizou-se o levantamento da história médica, atentando-se para as doenças geralmente associadas à DC, além de exame clínico para detectar manifestações bucais. Os resultados demonstraram a associação das alterações bucais com a DC, registrando-se dor ou ardência lingual (30\% do grupo DC e 10\% do grupo controle), além de lesões na mucosa oral, eritema ou ulceração, localizadas nos lábios, palato, mucosa ou língua (55\% do grupo DC e $23 \%$ do grupo controle). As úlceras foram o tipo mais comum de lesão oral, apresentando-se sob a forma de púrpura, papular ou erosiva, geralmente com a margem eritematosa.

Estes resultados mostram a importância de reconhecer lesões bucais típicas, desenvolvidas geralmente por deficiências nutricionais, como indícios de que o indivíduo é celíaco.

\section{O N CLUSÃ O}

Apesar de não haver dados estatísticos sobre a prevalência de DC no Brasil, a mesma vem crescendo. 
A DC apresenta várias formas clínicas e, nos últimos tempos, as mais comuns são as formas atípicas, cujos sintomas geralmente passam despercebidos. Dentre os principais sintomas, temos a anemia por deficiência de ferro (refratária à ferroterapia oral), além de artrites, osteoporose, esterilidade, constipação intestinal, retardo no crescimento e hipoplasia do esmalte dentário.

O diagnóstico da DC é desafiador, pois as formas clínicas da doença vêm se modificando e, cada vez mais, são latentes ou assintomáticas. Portanto, percebê-las exige o envolvimento, não somente do gastroenterologista, mas também, de vários outros profissionais da saúde.

A maior incidência de sinais e sintomas bucais (hipoplasia do esmalte dental, úlceras na mucosa bucal, dor ou ardência lingual), em pacientes celíacos, mostra a fundamental importância de reconhecer essas alterações como auxiliares no diagnóstico desta enteropatia, já que, muitas vezes, esses são os únicos sinais clínicos de uma doença que, se não tratada, mais tarde pode levar a complicações, tais como deficiências nutricionais e, até mesmo, malignidade.

\section{REFERÊ NCIAS}

1. Walker SJ. Celiac Disease. In: Walker W. Pediatric gastrointestinal disease: Pathophysiology, diagnosis, management. 2nd ed. St. Louis: Mosby; 1996. p.841-61.

2. Kotze LMS. Distúrbios entéricos da absorção. In: Dani R. Gastroenterologia essencial. Rio de Janeiro: Guanabara Koogan; 1998. p.211-24.

3. Guevara GP. Enfermedad celíaca. Rev Chil Pediatr. 2002; 73(4):394-7.

4. Landaburo RV, Pérez FS. Celiaquía: nuevos rostros de una antigua enfermedad. Medicentro 2002; $6(2)$.

5. Catassi C, Ratsch IM, Fabiani E, Rossini M, Bordicchia F, Candela F, et al. Coeliac disease in the year 2000: exploring the iceberg. Lancet. 1994; 343(8891):200-3.

6. Wetiz JCV, Montalva RD, Alarcón TO, Contreras LM. Determinación de anticuerpos anti-transglutaminasa en el diagnóstico de enfermedad celíaca. Rev Méd Chile. 2003; 131(1):25-9.
7. Romaldini CC, Barbieri D. Anticorpos séricos na doença celíaca. Arq Gastroenterol. 1999; 36(4): 258-64.

8. Roessler JL, Ríos GM, Alarcón TO, Bergenfreid CO, Moudragón AO, Araya MQ. Enfermedad celíaca en el adolescente y adulto joven. Un desafío para gastroenterólogos de niños y adultos. Rev Méd Chile. 2001; 129(7):743-8.

9. Sdepanian VL, Morais MB, Neto UF. Doença celíaca: a evolução dos conhecimentos desde sua centenária descrição até os dias atuais. Arq Gastroenterol. 1999; 36(4):244-57.

10. Morais MB, Sdepanian VL, Neto UF. Doença Celíaca. Nutr Pauta. 2001; 51:30-4.

11. Sdepanian VL, Scaletsky ICA, Morais MB, Neto UF. Pesquisa de gliadina em medicamentos - informação relevante para a orientação de pacientes com doença celíaca. Arq Gastroenterol. 2001; 38(3):176-82.

12. Sdepanian VL, Morais MB, Neto UF. Doença celíaca: avaliação da obediência à dieta isenta de glúten e do conhecimento da doença pelos pacientes cadastrados na Associação dos Celíacos do Brasil. Arq Gastroenterol. 2001; 38 (4):232-9.

13. Santos CHS, Almeida ILB, Gomes MDC, Serafim A, Pereira MM, Muszkat $M$, et al. Calcificação intracraniana occipital bilateral, epilepsia e doença celíaca. Arq Neuro-Psiquiatr. 2002; 60(3-B): 840-3.

14. Holmes GKT. Non-malignant complications of coeliac disease. Acta Paediatr. 1996; 85(412):68.

15. Cataldo F, Marino V, Bottaro G, Greco P, Ventura A. Celiac disease and seletive immunoglobulin a deficiency. J Pediatr. 1997; 131(2):306.

16. Collin P, Salmi J, Haellstroem O, Reunala T, Pasternack A. Autoimmune thyroid disorders and coeliac disease. Eur J Endocrinol. 1994; 130:7.

17. Madrid RB, et al. Complicación asociada a la enfermedad celíaca. Med Interna. 2002; 19:81-4.

18. Ferguson A, Kingstone K. Coeliac disease and malignancies. Acta Paediatr. 1996; 85(412):78.

19. Sdepanian VL, Morais MB, Neto UF. Doença celíaca: características clínicas e métodos utilizados no diagnóstico de pacientes cadastrados na Associação dos Celíacos do Brasil. J Pediatr. 2001; 77(2):131-8.

20. Kotze LMS, Utiyama SRR, Nisihara RM, Mocelin V, Carvalho RFA, Zeni MPB, et al. Comparação dos anticorpos anti-reticulina e antiendomísio classe IgA para diagnóstico e controle da dieta na doença celíaca. Arq Gastroenterol 1999; 36(4):177-84.

21. Lähteenoja $H$, Toivaner $A$, Viander $M$, Maki M, Irjala $\mathrm{K}$, Raiha I, et al. Oral mucosal changes in coeliac 
patients on a gluten-free diet. Eur J Oral Sci. 1998; 106(5):899-906.

22. Aine L. Permanent tooth dental enamel defects leading to the diagnosis of coeliac disease. Br Dent J. 1994; 177(7):254-5.

23. Aine L. Coeliac-type permanent-tooth enamel defects. Ann Med. 1996; 28(1):9-12.

24. Nikiforuk G, Frase D. The etiology of enamel hypoplasia: a unifying concept. J Pediatr. 1981; 98(6):888-93.

25. Aguirre JM, Rodríguez R, Oribe D, Vitoria JC. Dental enamel defects in celiac patients. Oral Surg Oral Med Oral Path Oral Radiol Endod. 1997; 84(6):646-50.

26. Aine L. Dental enamel defects and dental maturity in children and adolescents with coeliac disease. Proc Finn Dent Soc. 1986; 82(3):71.

27. Aine L, Maki M, Collin P, Keyrilainen O. Dental enamel defects in celiac disease. J Oral Pathol Med. 1990; 19(6):241-5.
28. Mariani P, Mazzilli MC, Margutti G, Lionetti P, Triglione $\mathrm{P}$, Petronzelli $\mathrm{F}$, et al. Coeliac disease, enamel defects and HLA typing. Acta Paediatr. 1994; 83(12):1272-5.

29. Smith DMH, Miller J. Gastro-enteritis, coeliac disease and enamel hypoplasia. Br Dent J. 1979; 147:91-5.

30. Ballinger A, Hughes C, Kumar P, Hutchinson I, Clark $M$. Dental enamel defects in coeliac disease. Lancet. 1994; 343(22):230-1.

31. Seow WK. Enamel hypoplasia in the primary dentition: a review. J Dent Child. 1991; 58(6): 441-52.

32. Pinto GAC. Odontopediatria. 6a.ed. São Paulo: Santos; 1997. Cap. 2.

33. Maki M, Aine L, Lipsanen V, Koskimies S. Dental enamel defects in first- degree relatives of coeliac disease patients. Lancet. 1991; 337(8744):763-4.

Recebido para publicação em 29 de janeiro e aceito em 18 de maio de 2004. 